\title{
Galactic planetary nebulae with Wolf-Rayet nuclei
}

\section{A consistent observational data set $^{\star, \star \star}$}

\author{
M. Peña ${ }^{1}$, G. Stasińska ${ }^{2}$, and S. Medina ${ }^{1}$ \\ 1 Instituto de Astronomía, Universidad Nacional Autónoma de México, Apdo. Postal 70 264, México D.F. 04510, \\ México \\ e-mail: selene@astroscu.unam.mx \\ 2 DAEC, Observatoire de Paris-Meudon, 92195 Meudon Cedex, France \\ e-mail: grazyna.stasinska@obspm.fr
}

Received 21 September 2000 / Accepted 5 December 2000

\begin{abstract}
We present high resolution spectrophotometric data for a sample of 34 planetary nebulae with [WC] spectral type central stars (WRPNe) in our Galaxy. The observed objects cover a wide range in stellar characteristics: early and late [WC] type stars, as well as weak-emission line stars (WELS). Physical conditions in the nebulae (electron density and temperatures) have been obtained from various diagnostic line ratios, and chemical abundances have been derived with the usual empirical scheme. Expansion velocities were estimated in a consistent manner from the line profiles for most objects of the sample. A statistical study was developed for the derived data in order to find fundamental relationships casting some light on the evolutionary status of WRPNe. We found evidence for a strong electron temperature gradient in WRPNe which is related to nebular excitation. Such a gradient is not predicted in simple photoionization models. Abundance ratios indicate that there seems to be no preferential stellar mass for the Wolf-Rayet phenomenon to occur in the nucleus of a planetary nebula. Two objects, M 1-25 and M 1-32, were found to have a very small Ne/O ratio, a property difficult to understand. We reexamined the relation between the nebular properties of the WRPNe and the spectral types of the central stars. Our data confirm the trend found by other authors of the electron density decreasing with decreasing spectral type, which was interpreted as evidence that [WC] stars evolve from late to early [WC] types. On the other hand, our data on the expansion velocities do not show the increase of expansion velocity with decreasing spectral type, that one might expect in such a scenario. Two objects with very late [WC] type central stars, K 2-16 and PM 1-188, do not follow the general density sequence, being of very low density for their spectral types. We suggest that the stars either underwent a late helium flash (the "born again" scenario) or that they have had a particularly slow evolution from the AGB. The 6 WELS of our sample follow the same density vs. [WC]-type relation as the bona fide WRPNe, but they tend to have smaller expansion velocities. Considerations about the evolutionary status of WELS must await the constitution of a larger observational sample. The analysis of the differences between the WRPNe in the Magellanic Clouds (distribution of [WC] spectral types, N/O ratios) and in the Galaxy indicates that metallicity affects the $[\mathrm{WR}]$ phenomenon in central stars of planetary nebulae.
\end{abstract}

Key words. planetary nebulae: general - stars: Wolf-Rayet - planetary nebulae: individual: K 2-16, PM 1-188, M 1-32, M 3-15

\section{Introduction}

Since the pioneering work of Paczyński (1971), the basic scheme for the production of planetary nebulae (here-

Send offprint requests to: M. Peña, e-mail: miriam@astroscu.unam.mx

* Based on data obtained at the Observatorio Astronómico Nacional, SPM, B.C., México

** Tables 2 and 3 are only available at http://www.edpsciences.org inafter PNe) and the evolution of their nuclei is relatively well understood. Planetary nebulae are formed from stars of initial masses between 1 and $8 M_{\odot}$, which have completely burnt hydrogen and helium in their cores, have twice climbed the giant branch and lost most of their mass through intense winds. At this stage, the stars consist of dense carbon-oxygen cores of about $0.6 M_{\odot}$ surrounded by an envelope composed of two thin shells. The innermost one is composed of helium, and the outermost of hydrogen. When the strong winds cease, the stars evolve 
towards larger effective temperatures and start ionizing the matter lost previously in the winds. The physical reason for the departure from the asymptotic giant branch is not quite understood, nor is the nature of the energy source (hydrogen- or helium-burning) during the evolution of the planetary nebula nuclei (Iben 1995).

About 50 planetary nebulae in our Galaxy are known to have central stars of Wolf-Rayet (hereinafter [WR]) type among 350 PNe with a stellar continuum measured and over $1000 \mathrm{PNe}$ with nebular spectroscopy available (Tylenda et al. 1993; Górny \& Stasińska 1995). All these [WR] central stars have been reported to be of [WC] spectral type, mostly [WC 2-4] and [WC 8-11] (Tylenda et al. 1993) and their atmospheres are almost pure helium and carbon (e.g. Hamann 1997). These planetary nebulae with [WR] nuclei, hereinafter WRPNe, have recently been the subject of much attention (e.g. Górny \& Stasińska 1995; Crowther et al. 1998; Górny \& Tylenda 2000; Górny et al. 2000 ), as they pose a problem for the theory of the evolution of PN central stars. Indeed, helium-burning models (Wood \& Faulkner 1986; Vassiliadis \& Wood 1994) do maintain a thin hydrogen-rich outer layer, as emphasized by Górny \& Tylenda (2000). One way to obtain a hydrogen-free outer layer is through the born-again scenario (Iben et al. 1983), when a final helium-shell flash occurs while the star is in its cooling phase, and drives it back to the asymptotic giant branch (AGB). However, such a scenario cannot hold for all the WRPNe, as shown by Górny \& Tylenda (2000). They suggest that the central stars of most WRPNe evolve directly from the AGB. The high carbon abundance observed in these stars implies that deep mixing has occurred (Herwig et al. 1997; Herwig et al. 1998).

It has been suggested that the spectral sequence of [WC] stars corresponds to an evolutionary sequence from late to early types, ending with the PG 1159 type stars. This suggestion was based on the analysis of the stellar atmospheres of [WC] stars (Hamann 1997; Leuenhagen \& Hamann 1998) and on the nebular properties of the WRPNe (Acker et al. 1996). Górny \& Tylenda (2000) provided further observational arguments for the existence of such an evolutionary sequence.

Tylenda et al. (1993) defined a category of "weak emission line stars" (hereinafter WELS), whose emission lines have much lower equivalent widths than the bona fide [WR] stars. Parthasarathy et al. (1998) claim that WELS are an intermediate stage between [WC] and PG 1159 stars.

This, however, is not the end of the story. First, not all WRPNe belong to proposed evolutionary sequence. For example 4 WRPNe with late type central stars, namely M 4-18, He 2-459, He 2-99 and NGC 40, were considered to have too low densities for such a scenario, and it has been suggested that they may have a different evolutionary status $^{1}$. Second, the carbon abundance in the wind of [WC]

\footnotetext{
1 According to our data, NGC 40, M 4-18 and He 2-459 could actually belong to the sequence, see Sect. 4 .
}

stars decreases with decreasing spectral type (Hamann 1997), while the reverse is expected, at least from simple considerations. Third, Peña et al. (1998) and de Marco \& Crowther (1999) have shown that PNe with nuclei of same [WC] type may have very different nebular properties (morphologies, abundance ratios, etc.) suggesting that stars with quite different initial masses can pass through the same [WC] stage. Finally, the Wolf-Rayet phenomenon is known to be variable, at least in a few cases (Peña et al. 1997a; Werner et al. 1992). Thus, it is not obvious that the stars now appearing as [WC 2-3] were of [WC-late] type before.

In order to provide a homogeneous data set for studying the nature and evolution of WRPNe, in 1995 we started a program of systematic observations of WRPNe, obtaining high resolution spectroscopic data of the nebulae and their nuclei. One of the advantages of high resolution is to safely deblend the nebular and stellar lines. This is essential for compact nebulae surrounding late type stars, where the stellar lines might otherwise affect the nebular diagnostics. In addition, this allows to estimate expansion velocities of the nebular envelopes. The first results of this effort have been presented in Peña et al. (1998), where a sample of high excitation nebulae, ionized by [WC 2-3] central stars were analyzed by computing detailed photoionization models for each object. In this work, we report observational data for 29 additional PNe with nuclei of all spectral types, including a few WELS.

In Sect. 2, we present our sample, describe our observations of the nebulae and the central stars and derive the usual plasma diagnostics (electron densities and temperatures, abundance ratios). In Sect. 3, we discuss the systematics of the plasma properties in our sample. In Sect. 4 , we examine the relation between the plasma properties and the $[\mathrm{WC}]$ type of the central stars. In Sect. 5, we present and discuss the expansion velocities of our sample. The main conclusions of this work are summarized in Sect. 6 .

\section{Observations}

We selected for observation a sample of 23 WRPNe from the list of Tylenda et al. (1993). Together with the 5 WRPNe with early-type central stars presented in Peña et al. (1998), this constitutes a sample of 28 WRPNe.

We also observed 6 PNe ionized by WELS (NGC 6629, NGC 6578, NGC 6567, NGC 6543, IC 5217, Cn 2-1) from the list of Tylenda et al. (1993). WELS actually represent a mixed bag, since a few of them are known to present hydrogen features in their spectra (from our list these are NGC 6629 and NGC 6543, respectively classified as Of(H) and Of WR(H) by Méndez 1990). For simplicity, in the following, we will use the term WRPNe for all the PNe of our sample, regardless of whether they are excited by [WR] stars or WELS, unless explicitly stated.

Our sample is not a complete one, in that it does not reflect the true proportion of nuclei of various spectral types, and should not be used to infer such things as 
stellar lifetimes for example. The aim was rather to obtain a homogeneous data set for the entire range of [WC] spectral types, from [WC 11] to [WC 2].

The objects of our sample are presented in Table 1, which gives their PN G numbers from the StrasbourgESO catalogue (Acker et al. 1992) as well as their usual names.

Several observing runs have been performed at the Observatorio Astronómico Nacional, San Pedro Mártir, B.C., México, with the 2.1-m telescope and the REOSC echelle spectrograph in the high resolution mode. The general characteristics of the spectrograph have been reported by Levine \& Chakrabarty (1994). The spectral range covered is wide enough to obtain the main diagnostic line ratios and the spectral resolution is always better than $0.3 \AA$ per pixel. Table 1 shows the log of observations and describes the instrumental setup, the wavelength range and spectral resolution for each run.

Bright spectrophotometric standard stars from the list by Hamuy et al. (1992) were observed in all the runs for flux calibration. A Th-Ar lamp was used for wavelength calibration in all the spectral ranges and a tungsten bulb was used for flat-fielding. Data reduction was performed using the $\mathrm{IRAF}^{2}$ reduction package.

As Table 1 shows, for each PN, we have at least two consecutive observations in each run, with exposure times allowing a good signal-to-noise in the weak lines. In addition, for bright objects, short exposures were obtained in order to avoid saturation in the most intense lines. The exposure times vary from 2 to $15 \mathrm{~min}$, depending on the object. A number of $\mathrm{PNe}$ were observed at various epochs. The slit position was always centered at the central star except for some extended objects where several positions were observed. In this paper, we only present data for the central position, unless otherwise stated (see Table 2).

After extraction and calibration of the spectra, we found that the differences between spectra obtained in the same night, with the same slit dimensions, are smaller than $5 \%$ (under photometric conditions). Spectra from different observing runs however show larger differences. We estimated the errors in the line intensities from the measured signal-to-noise and the differences between the various observing runs.

\subsection{Stellar data and classification}

The spectral types of [WC] central stars are taken from recent literature and are listed in Table 2 (with references indicated in the footnotes). For WELS for which no spectral type was available, we assigned a tentative type using criteria based on the classification scheme of [WC] stars by van der Hucht et al. (1981), Méndez \& Niemela (1982) and $\mathrm{Hu} \&$ Bibo (1990). This scheme is based on the relative strength of the optical C IV 5805 and C III 5695 lines and the Ov 5598, O vi 5290 and O vir 5670 lines. Crowther

${ }^{2}$ IRAF is distributed by NOAO, which is operated by AURA, Inc., under contract with the NSF. et al. (1998) proposed a unified and more quantitative classification scheme of WC and WO stars, which is independent of the $\mathrm{C} / \mathrm{O}$ ratios in the star atmospheres but requires good signal-to-noise in the diagnostic lines. We considered the option of using this scheme for all our objects, but because of the high resolution of our spectra, which spreads the stellar features over many pixels, we were able to classify accurately only the stars brighter than about $m_{V}=16$. In any case, for those objects for which several classifications are available, the proposed spectral types differ by one unit at most. It must be recalled that any spectral classification of [WC] stars orders the objects with respect to one parameter only, basically the ionization structure of the atmosphere. This ionization structure depends primarily on the temperature of the star, but other parameters such as the wind density do play a role. So, even in the Crowther et al. (1998) classification scheme, two stars of different temperatures may have the same spectral type if they differ in the other wind properties.

We also list in Table 2 the stellar visual magnitudes $m_{V}$ derived from the stellar continuum fluxes at $5480 \AA$ measured in our spectra (after subtracting the nebular continuum using the expressions given by Pottasch 1984). For stars brighter than $m_{V}=15$, the uncertainties in our measurements are about \pm 0.3 mag considering that a small fraction of the light could have escaped from the narrow slit. Fainter magnitudes have much larger uncertainties. For the bright stars, our values are in general in agreement with the stellar magnitudes reported in Acker et al. (1992). There are however some discrepancies larger than 0.5 mag. For instance, in our data Cn 1-5 is fainter, NGC 6567 is brighter, NGC 6551 is brighter, K 2-16 is fainter, etc., which are possibly indicating that stars could be variable. Our present data do not allow us to analyze the origin of the discrepancies or the possible stellar variations. Better photometric data are required for such an analysis.

\subsection{Nebular data}

The observed calibrated fluxes were corrected for reddening according to the expression:

$\log I(\lambda) / I(\mathrm{H} \beta)=\log F(\lambda) / F(\mathrm{H} \beta)+c(\mathrm{H} \beta) \times f_{\lambda}$

where $I(\lambda)$ and $F(\lambda)$ are the dereddened and observed fluxes respectively, $c(\mathrm{H} \beta)$ is the logarithmic reddening correction at $\mathrm{H} \beta$, and $f_{\lambda}$ is the reddening law. We employed the reddening law by Seaton (1979). The value of $c(\mathrm{H} \beta)$ was derived for each object from the Balmer decrement, by considering case B recombination theory (Hummer \& Storey 1987).

Dereddened intensities for the most important lines, relative to $\mathrm{H} \beta$, are listed in Table 2 . The 5 objects already presented in Peña et al. (1998) are also listed in Table 2, where additional data not reported before were included.

The intensities of the strong lines (observed flux larger than $\sim 10^{-14}$ erg $\mathrm{cm}^{-2} \mathrm{~s}^{-1}$ ) are generally 
Table 1. Log of observations ${ }^{(1)}$

\begin{tabular}{|c|c|c|}
\hline $\mathrm{PN} \mathrm{G}$ & Main Name & Observing dates (exposure times in minutes) \\
\hline $001.5-6.7$ & SwSt 1 & $970804(15,10,5)$ \\
\hline $002.4+5.8$ & NGC 6369 & $950729(15,15), 950731(15,15), 960615(15,15,15)$ \\
\hline $002.2-9.4$ & Cn 1-5 & $960617(15,15,15)$ \\
\hline $003.1+2.9$ & $\mathrm{Hb} 4$ & $960614(15,10,10,10,15)$ \\
\hline $004.9+4.9$ & M 1-25 & $960617(15,15,15)$ \\
\hline $006.8+4.1$ & M 3-15 & $960617(15,15,15)$ \\
\hline 009.4-5.0 & NGC 6629 & $970804(10,15)$ \\
\hline $010.8-1.8$ & NGC 6578 & $960614(15,15,15)$ \\
\hline $011.9+4.2$ & M 1-32 & $960614(15,15,15)$ \\
\hline 011.7-0.6 & NGC 6567 & $970804(10,10)$ \\
\hline $012.2+4.9$ & PM 1-188 & $970803(15,15)$ \\
\hline $017.9-4.8$ & M 3-30 & $960617(15,15)$ \\
\hline $027.6+4.2$ & M 2-43 & $970617(15,15,15)$ \\
\hline $029.2-5$. & NGC 6751 & $970803(15,15)$ \\
\hline $048.7+1.9$ & He $2-429$ & $991004(15,15)$ \\
\hline 061.4-9.5 & NGC6905 & $950729(15,15,15,15), 950731(15,15,15), 960614(15,15,10,10)$ \\
\hline $064.7+5.0$ & $\mathrm{BD}+303639$ & $970804(10,5,2), 991004(2,2,1.5)$ \\
\hline $068.3-2.7$ & He $2-459$ & $970803(15,15), 991004(15,15)$ \\
\hline $089.0+0.3$ & NGC 7026 & $950730(10,15,10,1), 950731(15,15,15), 981213(15,8)$ \\
\hline $096.4+29.9$ & NGC 6543 & $950730(2,10,10,2), 950731(10,10,10), 960615(2,2,4,4)$ \\
\hline $096.3+2.3$ & K $3-61$ & $991004(15,15)$ \\
\hline $100.6-5.4$ & IC 5217 & $991004(10,5)$ \\
\hline $120.0+9.8$ & NGC 40 & $981212(15,15)$ \\
\hline $130.2+1.3$ & IC 1747 & $950730(15), 950731(15,5), 981213(1,15)$ \\
\hline $144.5+6.5$ & NGC 1501 & $981213(15,15), 991004(15,15)$ \\
\hline $146.7+7.6$ & M 4-18 & $981213(15,15)$ \\
\hline $161.2-14.8$ & IC 2003 & $991004(10,10,7)$ \\
\hline $189.1+19.8$ & NGC 2371-72 & $981214(15,15,15,15)$ \\
\hline $243.3-1.0$ & NGC $2452^{(2)}$ & $981212(15,15), 981213(15,15)$ \\
\hline $278.8+4.9$ & $\mathrm{~PB} 6^{(2)}$ & \\
\hline 278.1-5.9 & NGC $2867^{(2)}$ & \\
\hline $286.3+2.8$ & He $2-55^{(2)}$ & \\
\hline $352.9+11.4$ & K 2-16 & $960614(15,15,15), 970803(15,15)$ \\
\hline $356.2-4.4$ & Cn 2-1 & $970804(15,15)$ \\
\hline
\end{tabular}

${ }^{(1)}$ Set-up July 1995: $1024 \times 102423 \mu$ CCD, 3600-6700 $\AA$, slit $4^{\prime \prime} \times 13^{\prime \prime}$, res. $\sim 0.3 \AA$.

Set-up June 1996: $1024 \times 102423 \mu \mathrm{CCD}, 3500-6650 \AA$, slit $4^{\prime \prime} \times 13^{\prime \prime}$, res. $\sim 0.3 \AA$.

Set-up Aug. 1997: $2048 \times 204819 \mu \mathrm{CCD}, 3360-7360 \AA$, slit $4^{\prime \prime} \times 13^{\prime \prime}$, res. $\sim 0.2 \AA$.

Set-up Dec. 1998: $2048 \times 204819 \mu$ CCD, 3360-7360 $\AA$, slit $4^{\prime \prime} \times 13^{\prime \prime}$, res. $\sim 0.2 \AA$.

Set-up Oct. 1999: $2048 \times 204819 \mu \mathrm{CCD}, 3360-7360 \AA$, slit $4^{\prime \prime} \times 13^{\prime \prime}$, res. $\sim 0.2 \AA$.

(2) Objects observed at CTIO 1994/12/30; see Peña et al. (1998).

accurate within $10 \%$. Those with observed fluxes in the range $1-510^{-15} \mathrm{erg} \mathrm{cm}^{-2} \mathrm{~s}^{-1}$ are accurate within 20$30 \%$. The data marked with a colon have uncertainties larger than $50 \%$. The line ratios that are used to derive electron temperatures and densities are explicitly listed at the bottom of Table 2, together with their uncertainties.
Table 2 also gives the values of $c(\mathrm{H} \beta)$ derived from our observations and the observed fluxes in $\mathrm{H} \beta$. These $\mathrm{H} \beta$ fluxes are not of photometric quality but are given as an indication of the brightness of the objects. We also list in Table 2 the nebular diameters, taken from the StrasbourgESO PN catalogue (Acker et al. 1992). 


\subsection{Plasma diagnostics}

The plasma diagnostics were performed from the emission line ratios in a standard way, using the same atomic data as listed in Stasińska \& Leitherer (1996).

The wide wavelength range of our observations allowed us to determine the electron densities and electron temperatures from several line ratios.

Electron densities were derived from [O II] 3726/3729, [S II] 6717/6731 and [Ar IV] 4711/4740, electron temperatures from [O III] 4363/5007 and [N II] 5755/6583. The densities used in the derivation of the electron temperatures were those deduced from [O II] 3726/3729, when available, [S II] 6717/6731 otherwise. At the densities encountered in our sample, only the [N II] $5755 / 6583$ temperature is dependent on the densities, and only to a small amount.

The derived electron temperatures and densities are listed in Table 3, together with the error bars based on the uncertainties in the line ratios given in Table 2 .

Ionic abundances were then obtained, using $T_{\mathrm{e}}[\mathrm{O}$ III $]$ for the high ionization species and $T_{\mathrm{e}}[\mathrm{N}$ II] (when available) for the low ionization ones. All the ionic abundances were computed using the electron densities derived from [O II] 3726/3729 $\left(n_{\mathrm{e}}\right.$ from [S II] $6717 / 6731$ was used when the former was not available). The value of the density is not important for most of the ions we are interested in, except $\mathrm{O}^{+}$.

Elemental abundance ratios were computed from the ionic abundance ratios using the ionization correction factors of Kingsburgh \& Barlow (1994) (note that these ionization correction factors are in reasonable agreement with the modeling performed for the highly ionized objects studied in Peña et al. 1998). The abundance ratios $\mathrm{He} / \mathrm{H}$, $\mathrm{O} / \mathrm{H}, \mathrm{N} / \mathrm{O}$ and $\mathrm{Ne} / \mathrm{O}$ are given at the bottom of Table 3. The error bars that are listed for $\mathrm{O}, \mathrm{N}$ and $\mathrm{Ne}$ only take into account the uncertainties propagated from the uncertainties in the physical conditions (electron temperature and density). Uncertainties in the ionization correction factors are not included. It must be kept in mind that, in the case of low excitation objects, evidently for Sw St 1, BD+30 3639, He 2-459, and NGC 40, the helium abundances derived in such a way are merely lower limits, since neutral helium is not taken into account. It is only with a detailed photoionization modeling that one could put stronger constraints on the helium abundance in these objects, an attempt which is outside the scope of this paper.

\section{Systematics of nebular properties in our WRPNe sample}

\subsection{Density and temperature structure}

In this section, we compare the different density and temperature diagnostics, with the aim of looking for systematic trends. $\mathrm{PNe}$ are ordered as a function of $\mathrm{O}^{++} / \mathrm{O}^{+}$, a parameter which provides an easy description of the "excitation" of the nebula and is available for most of the objects of our sample. The few exceptions are PM 1-188, He 2-459, K 3-61, NGC 1501 and M 4-18.

Figure 1a shows the ratio $n_{\mathrm{e}[\mathrm{S} \text { II }]} / n_{\mathrm{e}[\mathrm{O} \text { II }]}$ as a function of $\mathrm{O}^{++} / \mathrm{O}^{+}$. PNe with $[\mathrm{WC}]$ central stars are represented by filled circles, PNe around WELS are represented by open circles (as will be the case for all our observational diagrams). Although the two determinations of $n_{\mathrm{e}[\mathrm{S} \mathrm{II}]}$ and $n_{\mathrm{e}[\mathrm{O} \text { II }}$ are fairly similar, the diagram shows a tendency for $n_{\mathrm{e}[\mathrm{S} \text { II }]} / n_{\mathrm{e}[\mathrm{O} \text { II] }}$ to be larger than one for WRPNe of low excitation, and marginally equal to one for WRPNe of high excitation. This tendency is the same if we use the [S II] collision strengths from Keenan et al. (1996) instead of those from Cai \& Pradhan (1993) that were used here.

In principle, one expects [S II] lines to be emitted in regions of lower ionization than [O II] lines, and this is confirmed by photoionization models. One explanation of this trend could be that, for low excitation nebulae at least, which are probably ionization bounded, the density is increasing outwards, towards the ionization front. For density bounded nebulae (as is probably the case for many high excitation objects), the emission in the [S II] and [O II] lines is largely due to trace ions present in the $\mathrm{O}^{++}$zone, and the $[\mathrm{S} \mathrm{II}]$ and $[\mathrm{O} \mathrm{II}]$ densities are expected to be equal.

Figure $1 \mathrm{~b}$ shows $n_{\mathrm{e}[\mathrm{ArIV}]} / n_{\mathrm{e}[\mathrm{O} \text { II] }}$ as a function of $\mathrm{O}^{++} / \mathrm{O}^{+}$. In the nebulae where $n_{\mathrm{e}[\mathrm{Ar} \text { Iv] }}$ can be derived, i.e. in the high excitation ones only, this ratio is generally compatible with 1 and, statistically, indicates no tendency for a density gradient.

It would be interesting to have a control sample of nonWRPNe observed with the same equipment to see whether ordinary PNe show a similar behavior.

Figure $1 \mathrm{c}$ shows the ratio $T_{\mathrm{e}}[\mathrm{O} \mathrm{III}] / T_{\mathrm{e}}[\mathrm{NII}]$ of the electron temperatures derived from the [O III $] 4363 / 5007$ and $[\mathrm{N} \mathrm{II}] 5755 / 6583$ ratios respectively, as a function of $\mathrm{O}^{++} / \mathrm{O}^{+}$. We note a tendency for $T_{\mathrm{e}}[\mathrm{O} \mathrm{III}] / T_{\mathrm{e}}[\mathrm{N}$ II $]$ to decrease as the excitation increases, with values significantly lower than 1 at the high excitation end.

Simple photoionization models at metallicities around half solar, typical of the objects of our sample (see next section), behave quite differently. $T_{\mathrm{e}}[\mathrm{O}$ III $] / T_{\mathrm{e}}[\mathrm{N}$ II $]$ increases steadily with stellar effective temperature as soon as $T_{*}$ becomes larger than $50000 \mathrm{~K}$, and exceeds one for $T_{*}>100000 \mathrm{~K}$. The reason why the electron temperature increases outwards at low $T_{*}$ is that the most energetic stellar photons are absorbed in the outer regions, and that the ions found there are less effective for the cooling. At high $T_{*}$, the energy gains are larger, inducing a higher electron temperature. Then, the ions in the outer zone become more effective for the cooling than the ions in the $\mathrm{O}^{++}$ zone, resulting in a negative outward temperature gradient in the nebula. In any case, the temperature gradient shown by the models is very mild. The $T_{\mathrm{e}}[\mathrm{O}$ III $] / T_{\mathrm{e}}[\mathrm{N} \mathrm{II}]$ versus $\mathrm{O}^{++} / \mathrm{O}^{+}$plot will be investigated further, both theoretically and observationally. We plan to obtain observations of a control sample of non-WRPNe with a similar equipment, to see if non-WR nebulae behave in a similar way (the publicly available data on large samples of PNe do not allow us to analyze this question with confidence, 

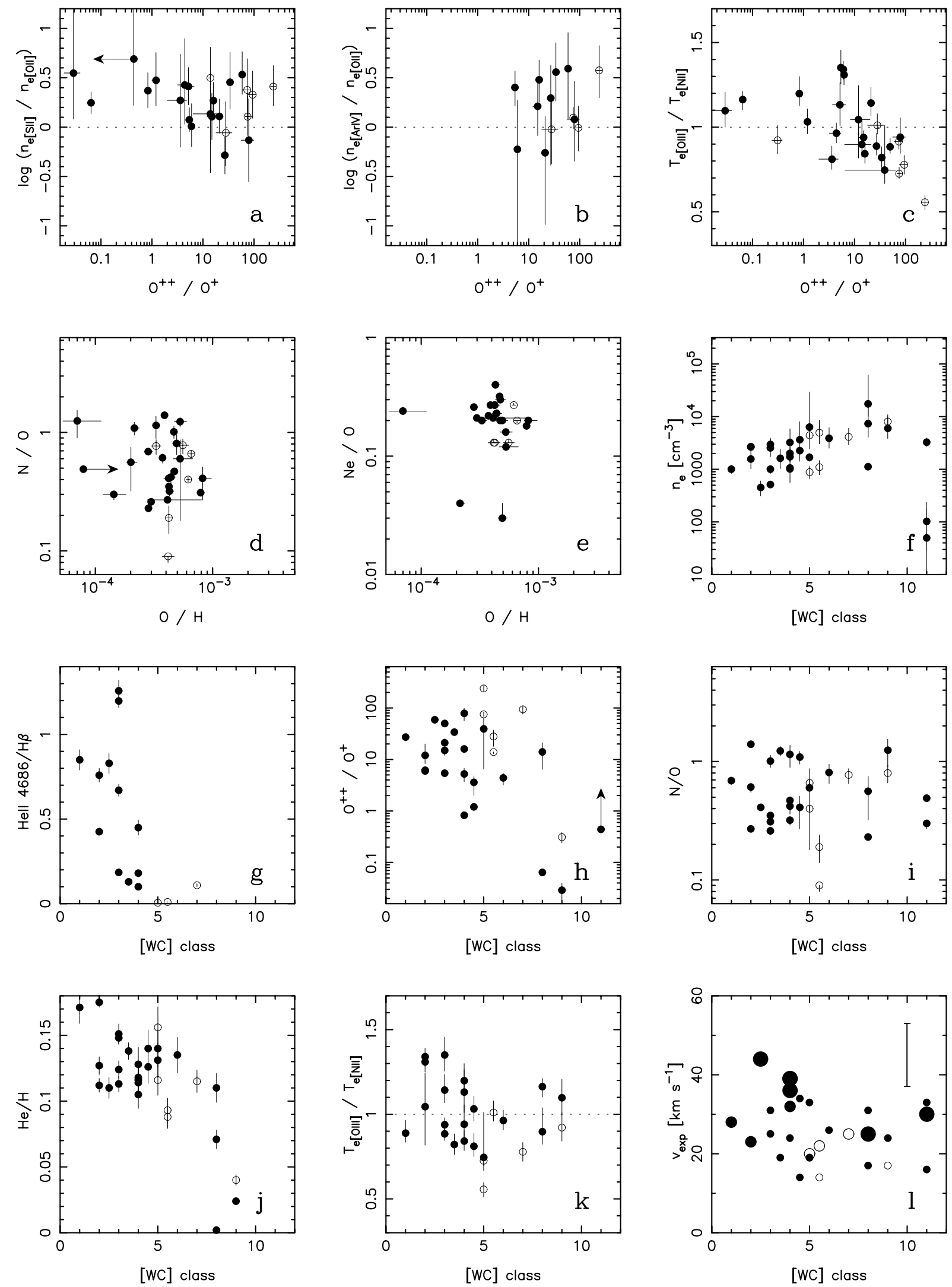

Fig. 1. The behavior of different nebular characteristics. Filled circles: PNe with [WC] nuclei, open circles: PNe with WEL stars. The $[\mathrm{WC}]$ types are defined with an uncertainty of one unit. The error bars in the computed quantities are indicated. In Panel l, we show the typical error bar corresponding to the measurement of the FWHM of the H $\beta$ line; large symbols indicate PNe with split line profiles; intermediate symbols, PNe with complex profiles and small symbols, PNe with Gaussian profiles 


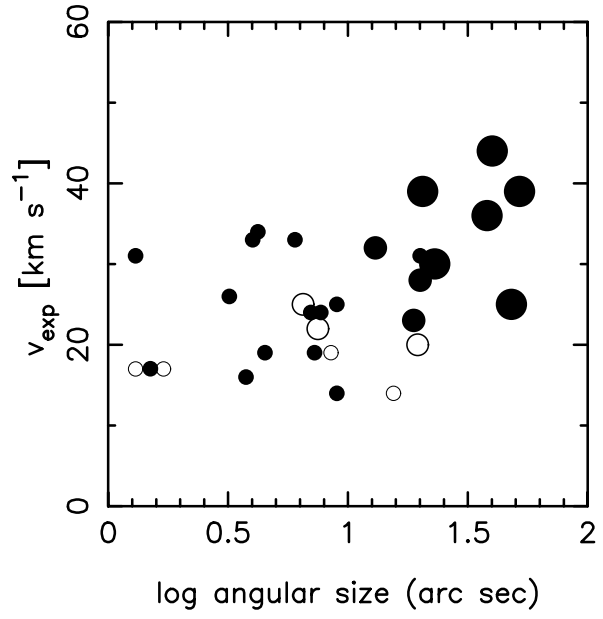

Fig. 2. The dependance of $v_{\exp }$ on the nebular diameter is shown. Symbols are the same as in Fig. 11

because $T_{\mathrm{e}}[\mathrm{O} \mathrm{III}]$ and $T_{\mathrm{e}}[\mathrm{N}$ II] are rarely available together and with good accuracy for the same object). For the moment, one can speculate that the low $T_{\mathrm{e}}[\mathrm{O}$ III $] / T_{\mathrm{e}}[\mathrm{N}$ II $]$ we see in our WRPNe sample could be due to a strongly inhomogeneous structure (but this does not seem supported by our measurements of the $n_{\mathrm{e}[\mathrm{Ar} \mathrm{IV}]} / n_{\mathrm{e}[\mathrm{O} \mathrm{II}]}$ ratio), or to additional heating by shocks or turbulence for example.

\subsection{Abundance ratios}

Regarding the $\mathrm{O} / \mathrm{H}$ ratios in our sample of WRPNe, we find that they spread over almost a factor 10 with a mean value $\sim 410^{-4}$. Such a distribution is comparable to the distribution found for example in the sample of PNe studied by Kingsburgh \& Barlow (1994), which contains only about $10 \%$ of WRPNe. One reason for such a large range is simply that the objects are located at various galactocentric distances, and that PNe are known to show an oxygen abundance gradient in the galaxy (Maciel \& Köppen 1994). That there is no significant difference in the $\mathrm{O} / \mathrm{H}$ distributions between our sample and that of Kingsburgh \& Barlow (1994) probably reflects the fact that the spatial distributions of WRPNe and ordinary PNe in the Galaxy are similar, as indicated by the similar distribution of coordinates and proper motions (Acker et al. 1996).

Diagrams relating abundance ratios in planetary nebulae are commonly used to cast some light on the nucleosynthesis and dredge-up processes in the progenitor stars (e.g. Henry 1990; Perinotto 1991; Groenewegen et al. 1995).

Figure 1d shows $\mathrm{N} / \mathrm{O}$ as a function of $\mathrm{O} / \mathrm{H}$ for our sample of WRPNe. A large spread in $\mathrm{N} / \mathrm{O}$ is seen, with values in N/O ranging over more than a factor 10. Such a spread is commonly observed in general samples of PNe (e.g. Torres-Peimbert \& Peimbert 1977; Kingsburgh \& Barlow 1994; Leisy \& Dennefeld 1996). It should be noted that $\mathrm{N} / \mathrm{O}$ is derived from the $[\mathrm{N} \mathrm{II}] 6584 /[\mathrm{O}$ II $] 3727$ intensity ratio, and is strongly affected by errors in the flux calibration, in the dereddening procedure and in the adopted electron densities. Nevertheless, the spread is much larger than the uncertainties. Some objects clearly show enhanced nitrogen abundances, which indicates that secondary nitrogen has been dredged-up to the surface in the central star progenitor. Others show quite modest $\mathrm{N} / \mathrm{O}$ ratios. One interpretation is that the progenitors of [WC] stars had quite different initial masses, similarly to the progenitors of the central stars of ordinary $\mathrm{PN}$, since the amount of dredged-up nitrogen in a post-AGB star depends on the progenitor's mass. However, reality could be more complex, as nitrogen yields depend also on the intensity of mass-loss on the AGB (Forestini \& Charbonnel 1997; van den Hoek \& Groenewegen 1997), and progenitors of [WC] central stars might experience different masslosses than progenitors of ordinary central stars.

Figure 1e shows $\mathrm{Ne} / \mathrm{O}$ as a function of $\mathrm{O} / \mathrm{H}$ in our sample of WRPNe. Compared to the N/O values, the Ne/O ones are much less scattered. This is also what is found in other samples of PNe (Henry 1990; Kingsburgh \& Barlow 1994; Stasińska et al. 1998) and is taken as evidence that oxygen and neon abundances are not modified by nucleosynthesis during the life of the progenitors. However, we note that two objects, whose neon abundances are measured for the first time (M 1-25 and M 1-32) have Ne/O smaller by about a factor 10 than the rest of our WRPNe (both these objects have $\mathrm{O}^{++} / \mathrm{O}^{+}>1$, therefore one does not expect the ionization correction factor to introduce a significant error in the determination of $\mathrm{Ne} / \mathrm{O}$ ). This is reminiscent of the planetary nebula $\mathrm{H} 4-1$ in the Galactic halo, which has an Ne/O ratio of $1.510^{-2}$ (Howard et al. 1997), and for which there seems to be no explanation so far. Note that, apart from their unusual Ne/O ratio, M 1-25 and M 1-32 do not stand out particularly in any of the diagram in Fig. 1, except that M 1-32 lies at the lower limit of expansion velocities (see Sect. 5 and Appendix).

\section{Is the proposed evolutionary [WC] sequence reflected in the nebular parameters?}

We now analyze the nebular parameters as a function of the spectral type of the nucleus (panels $f$ through $l$ in Fig. 1) ${ }^{3}$.

Figure 1f shows the electron density as a function of the [WC] spectral type. In this figure, the electron density is derived from the [O II] ratio (except in 7 cases where this ratio was not available and the density was derived from the $[\mathrm{S} \mathrm{II}])$. One expects $n_{\mathrm{e}[\mathrm{O} \mathrm{II}]}$ to be a better indicator of the overall nebular density than $n_{\mathrm{e}[\mathrm{S} \mathrm{II}]}$, since $\mathrm{S}^{+}$occupies a smaller volume than $\mathrm{O}^{+} . n_{\mathrm{e}[\mathrm{Ar} \text { Iv] }}$ would probably be even better, but this data is available only for a few objects. We find that the density decreases from late to early [WC] types. The slope is not as large as seen in the figure of Górny \& Tylenda (2000), who used the $n_{\mathrm{e}[\mathrm{S} \text { II] }}$ densities, but the trend is clearly there. In our sample, we do not

\footnotetext{
3 In panels f through 1, M 3-30, whose central star type is OVI, is represented with an abscissa of 1 . Cn 2-1, whose central star type is undetermined, is not represented.
} 
have many of the objects that, in the Górny \& Tylenda (2000) compilation, have $n_{\mathrm{e}[\mathrm{S} \mathrm{II}]}>10^{4} \mathrm{~cm}^{-3}$. Also, since we use $n_{\mathrm{e}[\mathrm{O} \text { II }]}$ rather than $n_{\mathrm{e}[\mathrm{SII}}, \mathrm{BD}+303639$ is plotted with a density smaller than $10^{4} \mathrm{~cm}^{-3}$.

Acker et al. (1996) and Górny \& Tylenda (2000) interpreted the decrease in electron density with decreasing [WC] spectral type as evidence for an evolutionary sequence from late to early type [WC]. Of the four objects that Górny \& Tylenda (2000) found to lie below the sequence (see the introduction), we have observations of NGC 40, M 4-18 and He 2-459. In our measurements, we find for He 2-459 a much larger density than used by these authors (17000 instead of $3600 \mathrm{~cm}^{-3}$ ), therefore this object is well within the sequence (see also Appendix). As for NGC 40 and M 4-18, they lie only marginally below the sequence.

On the other hand, two objects of [WC 11] type, K 2-16 and PM 1-188, which had no previous determination of the density, are clearly well below the sequence. These are then candidates for being PNe with central stars having experienced a late helium flash and returned to the AGB for a "born again" evolution. Alternatively, the stars may have been evolving very slowly off the AGB, either because of their low mass, or because of a very long transition time between the tip of the AGB and the beginning of the fast wind. Unfortunately, these objects are of very low excitation, so that there is only little relevant information in our spectra that could give some additional clues to their evolutionary status. PM 1-188 appears only in panels $\mathrm{f}$ and $\mathrm{l}$ of Fig. 1 . K 2-16 appears also in panels $\mathrm{a}, \mathrm{d}, \mathrm{h}$ and $\mathrm{i}$ (as a limit in the first three of them), and does not stand out particularly with respect to the other nebulae of our sample. In principle, it should be possible to distinguish between a "born again" scenario and one of slow stellar evolution. In the first case, the ionization stage of the nebula should be higher than the equilibrium value corresponding to the effective temperature of the central star, at least if the helium flash occurred recently (about a few hundred years ago). The spectra of K 2-16 and PM1-188 do not seem to indicate that the nebular gas is presently recombining from a more ionized stage, since no HeII lines are observed, favoring the hypothesis of a very slow evolution of the central star. However, a more careful theoretical and observational study of these objects would be warranted.

Note that, in Fig 1f, PNe that are ionized by WELS have intermediate densities as compared to the WRPN sample. If, as suggested by Parthasarathy et al. (1998), WELS were the descendents of [WC] early stars, they should be surrounded by more diluted nebulae. The fact that WELS are inside the [WC] sequence is intriguing. One explanation could be that [WC] and WELS are transient stages of the same stars.

Figure $1 \mathrm{~g}$ shows that the nebular He II 4686 line is seen only for early type [WC] stars, with the He II $4686 / \mathrm{H} \beta$ ratio tending to increase with decreasing spectral type. Qualitatively, this is expected, since spectral types are related with stellar effective temperatures. Differences in slit coverage, nebular masses, and stellar wind densities probably explain the scatter.

Figure 1 h shows $\mathrm{O}^{++} / \mathrm{O}^{+}$as a function of [WC] class. Only a weak trend is present. Actually, one does not expect a perfect correlation even between $\mathrm{O}^{++} / \mathrm{O}^{+}$and stellar effective temperature, since the stellar luminosity, the nebular mass, its average density and its density structure all affect the value of $\mathrm{O}^{++} / \mathrm{O}^{+}$. Therefore, the large dispersion seen in $\mathrm{O}^{++} / \mathrm{O}^{+}$at a given [WC] spectral type is not surprising.

Figure 1i shows N/O as a function of [WC] class. One can note the large scatter in $\mathrm{N} / \mathrm{O}$ at a given [WC] spectral type, reinforcing the suggestion that WRPNe have a range in progenitor masses.

Figure $1 \mathrm{j}$ displays $\mathrm{He} / \mathrm{H}$ as a function of [WC] class. As noted previously, the points with the lowest values of $\mathrm{He} / \mathrm{H}$ correspond to lower limits, since no account has been made for the presence of neutral helium to derive the total helium abundance. Figure $1 \mathrm{j}$ shows that these points represent objects with the latest, and therefore coolest [WC] central stars, which are indeed expected to produce a $\mathrm{He}^{+}$zone less extended than the $\mathrm{H}^{+}$zone. For intermediate and early type [WC] spectral classes, the helium abundances are reliable and we find a larger scatter than expected from observational uncertainties (which are of about $10-20 \%$ for each object). The contribution of the helium-rich wind from the [WC] star to the mean $\mathrm{He} / \mathrm{H}$ ratio observed in a nebula is expected to be rather small if we adopt a typical mass-loss rate of $10^{-6} M_{\odot} \mathrm{yr}^{-1}$ and a lifetime of $10000 \mathrm{yr}$. Therefore, the observed scatter in $\mathrm{He} / \mathrm{H}$ reflects differences in the chemical composition of the star atmospheres while on the AGB, likely due to differences in progenitors masses.

Figure $1 \mathrm{k}$ shows $T_{\mathrm{e}}[\mathrm{O}$ III $] / T_{\mathrm{e}}[\mathrm{N}$ II $]$ as a function of $[\mathrm{WC}]$ class. There is no clear trend in this figure, as compared with the trend seen in Fig. 1c. This seems to indicate that the behavior of $T_{\mathrm{e}}[\mathrm{O}$ III $] / T_{\mathrm{e}}[\mathrm{N}$ II $]$ has more to do with the nebular properties than with stellar properties or with an evolution scenario.

Interestingly, the WELS do not stand out with respect to the WRPNe in none of the diagrams discussed so far.

\section{The expansion velocities}

Velocity fields of planetary nebulae are an indicator of the genesis and the evolution of these objects (Mellema 2000). Basing on data compiled from the literature, it has been shown by Górny \& Stasińska (1995) that, on average, WRPNe have larger expansion velocities than nonWR PNe. This was interpreted as possibly due to the effect of a stronger stellar wind in the case of WRPNe.

The high spectral resolution of our observations allowed us to study the emission line profiles in most objects of our sample. A detailed description of the velocity fields, based on the study of the profiles for lines of various ions, will be given elsewhere (Medina et al., in preparation). Here we use a simple estimate of the expansion velocity, $v_{\text {exp }}$, derived from the $\mathrm{H} \beta$ profile, to see whether there 
is a correlation between expansion velocity and spectral type of the central star. Indeed, using expansion velocities compiled from the literature, Acker et al. (1996) found $v_{\text {exp }}$ to increase with decreasing [WC] type.

Deriving expansion velocities from line profiles is a notoriously difficult issue. The results depend on the adopted method (as shown by Gesicki \& Zijlstra 2000 using simple expanding photoionization models) and compiling expansion velocities from various sources may be misleading. Even observing all the objects with the same instrument and adopting the same procedure to derive the average expansion velocity, the result depends on the slit coverage and on the geometry of the source. In our objects we have found very different nebular line profiles. Several of them show split lines indicating the existence of a well defined shell. For such objects, the expansion velocity was assumed to be given by half the peak-to-peak distance in the $\mathrm{H} \beta$ profile. In other cases, only one resolved line component (roughly Gaussian) was found. In some objects, spatially resolved knots or condensations were seen. In all the cases of one component, the lines profiles were spectrally resolved, showing a FWHM larger than the instrumental resolution. The expansion velocity was then assumed to be given by the half width at half maximum (subtracting the instrumental width in quadrature). The expansion velocities derived in such a way for the objects of our sample are reported in Table 2, together with an indication of the shape of the line profile (split, Gaussian, complex).

Figure 11 displays $v_{\exp }$ as function of the [WC]-type. Objects with split lines are represented by the largest circles, objects with Gaussian profiles with the smallest circles. Complex profiles are indicated with intermediate circles. As in the preceding figures, filled circles are for true [WC], open circles are for WELS.

From this figure, the trend of $v_{\text {exp }}$ increasing with decreasing [WC] type relies on four objects (out of 29) which have the highest expansion velocities (NGC 6905, NGC 6751, NGC 1501 and NGC 6369). These four objects show split line profiles. Their larger measured expansion velocities may simply reflect an observational bias. This is confirmed by Fig. 2, which plots $v_{\exp }$ as a function of the apparent nebular diameter. It is clear, from this figure, that the type of line profile seen in our spectra depends on the fraction of the nebular image that is encompassed by the slit, and that the largest expansion velocities are found for the largest nebulae. From this, we conclude that there is no clear evidence of an increase in $v_{\text {exp }}$ with decreasing spectral type, contrary to what is reported by Acker et al. (1996). This weakens somehow the statement that most [WC] stars evolve from late to early types. Indeed, the naive expectation is that, in such a case, the strong winds from the star would produce an increase of the nebular expansion velocity with time. This, however, should be checked by detailed hydrodynamical models following the coupled stellar and nebular evolution on a sufficiently long timescale.
Interestingly, WELS show lower values of $v_{\exp }$ than bona fide WRPNe of similar angular size. Since the expansion velocity of a nebula depends on its entire former history, this fact tends to contradict the suggestion made in the previous section that WELS and [WC] stars might be transient stages of the same objects. The nature of WELS is by no means elucidated. As mentioned above, WELS probably represent a mixed bag. One obviously would need to observe a larger sample of these objects and to study simultaneously the stellar and nebular properties.

\section{Summary and prospects}

We have obtained high signal-to-noise high resolution spectra of a large sample of PNe with [WC] spectral type nuclei and a smaller sample of nebulae with weak emission line central stars. The nebulae are ionized by stars spanning the whole range of [WC] types, from [WC 2] to [WC 11]. These ample data, treated in a consistent way, enabled us to perform a significant statistical study of these objects. The main results are the following.

Concerning the plasma properties of the nebulae, we found a significant trend of the electron temperature ratio $T_{\mathrm{e}}[\mathrm{O} \mathrm{III}] / T_{\mathrm{e}}[\mathrm{N}$ II $]$ decreasing with increasing excitation of the object as measured by the $\mathrm{O}^{++} / \mathrm{O}^{+}$ratio. At the high excitation end, $T_{\mathrm{e}}\left[\mathrm{N}\right.$ II] is larger than $T_{\mathrm{e}}[\mathrm{O} \mathrm{III}]$ by several thousands degrees. This is not expected from simple photoionization models of planetary nebulae, and might indicate the presence of additional heating mechanisms in the outer zones (shocks or turbulence for example). Future observations of a control sample of non-WRPNe will enable us to show whether such a behavior is also seen in non-WR nebulae, giving some clues as to whether this additional heating mechanism can be due to the effect of the intense stellar winds from the central stars in the case of WRPNe.

The observed large dispersion in the $\mathrm{N} / \mathrm{O}$ ratios in WRPNe indicates that there is no preferential stellar mass for the Wolf-Rayet phenomenon to occur in the nucleus of a planetary nebula, confirming the finding by Górny \& Stasińska (1995).

Two objects, though, M 1-25 and M 1-32, whose neon abundances have been measured for the first time, have a $\mathrm{Ne} / \mathrm{O}$ ratio of the order of 0.03 , i.e. about ten times smaller than found in other PNe. There is only one PN known with a similarly low neon abundance: $\mathrm{H} 4-1$, in the Galactic halo (Howard et al. 1997). There is no explanation so far for such anomalous $\mathrm{Ne} / \mathrm{O}$ ratios in planetary nebulae.

Our data set enabled us to reexamine the relation between the nebular properties of the WRPNe and the spectral types of the central stars. It has been suggested that the $[\mathrm{WC}]$ sequence corresponds to an evolutionary sequence, from late to early type [WC]. Our plot of the electron density versus [WC] spectral type, obtained with a homogeneous data set, indicates that nebulae around [WC] early stars are more evolved than nebulae around [WC] late stars, seeming to confirm such a view. However, 
two objects with extremely late [WC] central stars (K 2-16 and PM 1-188) for which the electron densities have been determined for the first time, were shown to be of low density (well below $1000 \mathrm{~cm}^{-3}$ ). They may be similar in nature to He 2-99, which was not in our sample, but was suggested by Acker et al. (1996) to be powered by a star having experienced a late-helium flash. Alternatively, the central stars of these low density nebulae may have evolved on much larger time scales than the rest of $\mathrm{PN}$ nuclei, either due to their unusually small core mass or to an exceptionally long transition time from the tip of the AGB. Further observational and theoretical studies of these objects could enable one to distinguish between the various interpretations.

Our high resolution data allowed us to estimate the expansion velocities for almost all the PNe from our sample. Contrary to the claim by Acker et al. (1996), we do not find evidence for an increase in $v_{\exp }$ with decreasing spectral type. This, then, casts some doubt on the hypothesis that the [WC] stars evolve from late to early spectral type, since one would expect the expansion velocity to increase with time. However, a proper determination of the velocity fields in our planetary nebulae would require a more thorough analysis than performed here. Also, grids of detailed hydrodynamical models similar to the ones by Frank (1994) or Mellema (1994) but including cases of massive stellar winds would be necessary to test under what conditions exactly one expects the expansion velocities to increase with time.

Our sample includes 6 objects with weak emission line central stars (WELS). It has been suggested by Parthasarathy et al. (1998) that such stars are the evolved counterparts of early-[WC] stars (this, of course, cannot hold for the 2 WELS of our sample that have been reported to present clear hydrogen features). Most of our WELS are of intermediate [WC] types (when classified following criteria on intensity or equivalent width ratios of [WC] emission lines). In general, we find that the nebulae ionized by WELS follow the same tendencies as bona fide WRPNe. However, they show systematically lower expansion velocities than WRPNe of similar angular size (taking into account the angular size is important for the comparison of the expansion velocities, as we have shown). If one assumes that expansion velocities should be larger in the case of stronger central star winds, then the natural conclusion is that the WELS of our sample were not bona fide [WC] stars in the past. Such a conclusion is supported by the fact that the densities in nebulae ionized by WELS are not particularly low. Obviously, a much larger sample of WELS should be investigated before one can draw meaningful conclusions about the status of WELS.

It is interesting to compare the statistical properties of WRPNe in our Galaxy with those in the Magellanic Clouds (MC). There are four well established [WC] in the LMC (N 110, N 133, N 203 and MA 17) and two in the SMC (N 6 and Ln 302). This sample was studied in detail by Peña et al. (1997b).
The object SMC-Ln 302 is excited by a [WC 8] nucleus and, similarly to the galactic [WC 8] NGC 40, presents an electron density of about $1000 \mathrm{~cm}^{-3}$. That is, an apparently evolved nebula around a rather cool [WC]. All the other Magellanic Clouds [WC] stars have spectral types ranging from [WC 4] to [WC 7], and are surrounded by dense nebulae $\left(n_{\mathrm{e}[\mathrm{S} \text { II }]}>10^{4} \mathrm{~cm}^{-3}\right)$. In the Galactic sample, only half of the [WC] stars are in the [WC 4] to [WC 7] range (Tylenda et al. 1993; Górny \& Tylenda 2000) and their [S II] densities are mostly lower than $10^{4} \mathrm{~cm}^{-3}$.

Besides the striking difference concerning [WC] spectral type distributions and relations with nebular densities, another noticeable difference regards N/O. For the objects in the Magellanic Clouds, the $\mathrm{N} / \mathrm{O}$ ratios are found between 0.1 and 0.2 (excepting SMC-N6 which presents $\mathrm{N} / \mathrm{O}$ about 0.65 ), while for the objects in our Galactic sample they occupy a wide range of values and are all above $0.2(30 \%$ objects have $\mathrm{N} / \mathrm{O}>0.5)$. This offset in the distribution of $\mathrm{N} / \mathrm{O}$ ratios between the Magellanic Clouds and the Milky Way WRPNe is similar to the one found for non WRPNe (see Fig. 1 by Stasińska et al. 1998), where only the luminous $\mathrm{PNe}$ must be considered, since the WRPNe identified in the MC are all luminous). The fact that the distribution of N/O in MC WRPNe is extremely narrow could be an effect of small number statistics. In any case, the low N/O observed in the known WRPNe of the MCs seems to indicate that the $[\mathrm{WC}]$ progenitors did not undergo the second dredge-up event (although they obviously underwent a dredge-up phase that extracted large amounts of freshly-made carbon up to the stellar surface) implying that they possibly were not massive. This is at variance with the fact that the progenitors of Galactic WRPNe seem to have a large range of masses. Notoriously, the only known WRPN in the Magellanic Clouds with a probably high mass progenitor is LMC-N66, whose central star exhibits a highly variable mass loss, developing (apparently episodically) intense [WN]-early features (Peña et al. 1994; Peña et al. 1997a).

It seems clear that the differences in metallicity between the Magellanic Clouds and the Milky Way are largely affecting the $[\mathrm{WR}]$ phenomenon in central stars of planetary nebulae.

\section{Appendix: Notes on individual objects}

\section{K2-16}

K 2-16, ionized by a [WC 11] type star is a remarkable object. The central star is very bright with a visual magnitude of about 13 mag (probably variable). It was studied in detail by Leuenhagen et al. (1996) who derived a temperature $T_{*}=30000 \mathrm{~K}$, a terminal wind velocity of $300 \mathrm{~km} \mathrm{~s}^{-1}$, a mass loss rate of $4.410^{-7} M_{\odot} \mathrm{yr}^{-1}$ and a chemical composition (in mass fraction) $\beta_{\mathrm{H}} \leq 1 \%$, $\beta_{\mathrm{He}}=45 \%, \beta_{\mathrm{C}}=50 \%$ and $\beta_{\mathrm{O}}=5 \%$. That is, the star is showing helium burning products in the atmosphere. According to these authors, the star is very similar to 
other [WC 11] stars such as the nuclei of M 4-18, He 2-113 and others, with a similar evolutionary status.

On the other hand, the nebula in K 2-16 is quite different showing a very faint and extended shell with an angular diameter of about 20 arcsec. It presents a much lower density than nebulae around most of [WC]-late stars which are usually compact and very dense, and the shell shows a large expansion velocity.

In our spectra from the central zone, the star dominates the emission and its numerous and intense lines are blended with the faint nebular lines, hiding them. Therefore we extracted the nebular spectrum from the zone immediately outside the stellar continuum.

The oxygen abundance determined for this object is only a lower limit due to the faintness of [N II] 5755 for which only an upper limit is given.

\section{He 2-459}

This nebula, which has a [WC 8] central star, was one of those which were considered by Acker et al. (1996) as candidates for the "born again" scenario, since its [S II] ratio indicated a density of $3600 \mathrm{~cm}^{3}$, lower than in other nebulae surounding stars of similar [WC] type. Our observations from different observing runs consistently indicate a [S II] density of $17000 \mathrm{~cm}^{3}$. In this object, which is highly reddened, the $[\mathrm{OII}]$ ratio is not available. Interestingly, this object shows an extremely weak He I $\lambda 5876$ emission line (about 0.005 of $\mathrm{H} \beta$ ). The nebula is also of very low excitation, with a [O III] $\lambda 5007$ intensity of 0.03 of $\mathrm{H} \beta$. This is quite unexpected for a nebula excited by a [WC 8] central star, for which Leuenhagen \& Hamann (1998) have derived an effective temperature of $77000 \mathrm{~K}$, from a stellar atmosphere analysis. The H I, He I and He II Zanstra temperatures derived by these authors for their models of the star are 47000,45000 and $16000 \mathrm{~K}$ respectively. Could it be that, because of the very large mass loss rate in this $\operatorname{star}\left(10^{-5} M_{\odot} \mathrm{yr}^{-1}\right.$ assuming $\log L / L_{\odot}=3.70$, according to Leuenhagen \& Hamann 1998) and the large helium abundance in the atmosphere, the radiation shortward of the $24.6 \mathrm{eV}$ would be much more efficiently blocked that predicted? In any case, He 2-459 deserves a much more thorough theoretical and observational study.

\section{1-32 and M 3-15}

High velocity material has been detected in these objects. In both cases the line profiles show a strong narrow component with an almost unresolved expansion velocity lower than $20 \mathrm{~km} \mathrm{~s}^{-1}$ overimposed on faint blue and red wide wings. In the case of M 1-32, the wings extend to about $\pm 100 \mathrm{~km} \mathrm{~s}^{-1}$, while in M 3-15 the wings spread from $-80 \mathrm{~km} \mathrm{~s}^{-1}$ to $+90 \mathrm{~km} \mathrm{~s}^{-1}$. Only the HWHM of the narrow profiles have been used in Figs. 11 and 2 .

Another object where high velocity ejections have been detected is Hb 4. López et al. (1996) found collimated outflows, several arcsec away from the main body of this neb- ula with radial velocities of $\pm 150 \mathrm{~km} \mathrm{~s}^{-1}$. According to these authors, the nebula presents an expansion velocity of $21.5 \mathrm{~km} \mathrm{~s}^{-1}$, which is identical (within uncertainties) to our value from the HWHM profile.

The nature of the high velocity material in M 1-32 and M 3-15 could be different, as the emission comes from an unresolved central zone. Spatially resolved observations are required to elucidate where this high velocity emission comes from, but a bipolar or multipolar ejection cannot be excluded.

Acknowledgements. This work received partial support from the CNRS-CONACyT/México agreement (grant E130-983), CONACyT (grant 32594-E) and DGAPA/UNAM (grants IN-109696 and IN-100799), the University Paris 7 and the Observatoire de Paris-Meudon. MP thanks the DAEC and GS the UNAM for their hospitality.

\section{References}

Acker, A., Górny, S. K., \& Cuisinier, F. 1996, A\&A, 305, 944 Acker, A., Marcout, J., Ochsenbein, F., Stenholm, B., \& Tylenda, R. 1992, The Strasbourg-ESO Catalogue of Galactic Planetary Nebulae

Cai, W., \& Pradhan, A. K. 1993, ApJS, 88, 329

Chu, Y. H., Jacoby, G. H., \& Arendt, R. 1987, ApJS, 64, 529

Crowther, P. A., de Marco, O., \& Barlow, M. J. 1998, MNRAS, 296, 367

de Marco, O., \& Crowther, P. 1999, MNRAS, 306, 931

Forestini, M., \& Charbonnel, C. 1997, A\&AS, 123, 241

Frank, A. 1994, AJ, 107, 261

Gesicki, K., \& Zijlstra, A. A. 2000, A\&A, 358, 1058

Górny, S. K., \& Stasińska, G. 1995, A\&A, 303, 893

Górny, S. K., \& Tylenda, R. 2000, A\&A, in press

Górny, S. K., Stasińska, G., Szczerba, R., \& Tylenda, R. 2000, A\&A, submitted

Groenewegen, M. A. T., van den Hoek, L. B., \& de Jong, T. 1995, A\&A, 293, 381

Hamann, W.-R. 1997, Planetary Nebulae, IAU Symp. 180, ed. H. Habing, \& H. Lamers (Reidel, Kluwer), 91

Hamuy, M., Walker, A. R., Suntzeff, N. B., et al. 1992, PASP, 104,533

Henry, R. B. C. 1990, ApJ, 356, 229

Herwig, F., Blöcker, T., Schönberner, D., \& El Eid, M. 1997, A\&A, 324, 81

Herwig, F., Blöcker, T., \& Schönberner, D. 1998, A\&A, 340, 43

Howard, J. W., Henry, R. B. C., \& McCartney, S. 1997, MNRAS, 284, 465

Hu, J. Y., \& Bibo, E. A. 1990, A\&A, 234, 435

Hummer, D. G., \& Storey, P. J. 1987, MNRAS, 224, 609

Iben, I. 1995, Phys. Reports 250, 1

Iben, I., Kaler, J. B., Truran, J. W., \& Renzini, A. 1983, ApJ, 264,605

Keenan, F. P., Aller, L. H., Bell, K. L., et al. 1996, MNRAS, 281,1073

Kingsburgh, R. L., \& Barlow, M. J. 1994, MNRAS, 271, 257

Koesterke, L., \& Hamann, W.-R. 1997a, Planetary Nebulae, IAU Symp., 180, ed. H. Habing, \& H. Lamers (Reidel, Kluwer), 114

Koesterke, L., \& Hamann, W.-R. 1997b, A\&A, 320, 91 
Levine, S., \& Chakrabarty, D. 1994, Technical Report MU94-04, Instituto de Astronomía, Universidad Nacional Autónoma de México

Leuenhagen, U., \& Hamann, W.-R. 1998, A\&A, 330, 265

Leuenhagen, U., Hamann, W.-R., \& Jeffery, S. 1996, A\&A, 312,167

Leisy, P., \& Dennefeld, M. 1996, A\&AS, 116, 95

López, J. A., Steffen, W., \& Meaburn, J. 1996, ApJ, 485, 697

Maciel, W., \& Köppen, J. 1994, A\&A, 282, 436

Mellema, G. 2000, Low mass Wolf-Rayet Stars: origin and evolution, ed. L. B. F. M. Waters, \& A. A. Zijlstra, Ap\&SpSci, in press

Mellema, G. 1994, A\&A, 290, 915

Méndez, R. 1990, Stellar Atmospheres: Beyond Classical Models, Proc. of the Advanced Research Workshop, Trieste, Italy (Reidel, Dordrecht), 331

Méndez, R. H., \& Niemela, V. 1982, IAU Symp. 99, WolfRayet stars: Observations, Physics, Evolution, ed. C. W. H. de Loore, \& A. J. Willis (Dordrecht, Reidel), 457

Paczyński, B. 1971, Acta Astron., 21, 417

Parthasarathy, M., Acker, A., \& Stenholm, B. 1998, A\&A, 329, L9

Peña, M., Torres-Peimbert, S., Peimbert, M., Ruiz, M. T., \& Maza, J. 1994, ApJ, 428, L9

Peña, M., Hamann, W.-R., Koesterke, L., et al. 1997a, ApJ, 491,233
Peña, M., Ruiz, M. T., \& Torres-Peimbert, S. 1997b, A\&A, 324,674

Peña, M., Stasińska, G., Esteban, C., et al. 1998, A\&A, 337, 866

Perinotto, M. 1991, ApJS, 76, 687

Pottasch, S. R. 1984, Planetary Nebulae: A Study of Late Stages of Stellar Evolution, Astrophysics and Space Science, vol. 107 (Reidel, Dordrecht)

Seaton, M. 1979, MNRAS, 185, 57p

Schwarz, H. E., Corradi, R. L. M., \& Melnick, J. 1992, A\&AS, 94, 399

Stasińska, G., \& Leitherer, K. 1996, ApJS, 107, 661

Stasińska, G., Richer, M., \& McCall, M. 1998, A\&A, 336, 667

Torres-Peimbert, S., \& Peimbert, M. 1977, Rev. Mex. A\&A, 2, 181

Tylenda, R., Acker, A., \& Stenholm, B. 1993, A\&AS, 102, 595

van den Hoek, L. B., \& Groenewegen, M. A. T. 1997, A\&AS, 123,305

van der Hucht, K. A., Conti, P. S., Lundstrom, I., \& Stenholm, B. 1981, Space Sci. Rev. 28, 227

Vassiliadis, E., \& Wood, P. R. 1994, ApJS, 92, 125

Werner, K., Hamann, W.-R., Heber, U., et al. 1992, A\&A, 259, L69

Wood, P. R., \& Faulkner, D. J. 1986, ApJ, 307, 659 